\title{
O ESTILO DE LIDERANÇA EXERCIDO PELO ENFERMEIRO DE UNIDADE DE INTERNAÇÃO CIRÚRGICA SOB O ENFOQUE DA LIDERANÇA SITUACIONAL
}

Cristina Maria Galvão * Maria Auxiliadora Trevizan ** Namie Okino Sawada*

Neide Fávero*

GALVÃO, C.M.; TREVIZAN, M.A.; SAWADA, N.O.; FÁVERO, N. O estilo de liderança exercido pelo enfermeiro de unidade de internação cirúrgica sob o enfoque da liderança situacional. Rev.latino-am.enfermagem, Ribeirão Preto, v. 5, n. 2, p. 39-47, abril 1997.

A presente investigação orientou-se para a temática liderança enfocando o enfermeiro de unidade de internação cirúrgica. Como referencial teórico utilizamos o modelo de liderança proposto por Hersey e Blanchard, denominado Liderança Situacional. $O$ objetivo deste estudo consistiu em analisar a correspondência de opinião entre o enfermeiro e o pessoal auxiliar de enfermagem sobre o estilo de liderança exercido pelo enfermeiro de unidade de internação cirúrgica em relação às seis categorias de atividades assistenciais estudadas. Constatamos que os enfermeiros, nos dois hospitais investigados, adotaram prioritariamente, com o pessoal auxiliar estilos de liderança mais diretivos, ou seja, $E_{2}$ (persuadir) ou $E_{1}$ (determinar).

UNITERMOS: enfermeiro, unidade de internação cirúrgica, liderança situacional

\section{INTRODUÇÃO}

No contexto da Administração, à partir da Teoria das Relações Humanas, a incorporação de conhecimentos oriundos das ciências comportamentais representou um grande avanço nesta área. Nesse sentido, de acordo com CHIAVENATO $^{1}$, a liderança tornou-se um dos temas gerenciais mais pesquisado e estudado nos últimos cinquenta anos.

Para TANNENBAUM et al. ${ }^{9}$ a liderança é definida como "influência interpessoal exercida numa situação por intermédio do processo de comunicação, para que seja atingida uma meta ou metas específicas".

$\mathrm{Na}$ enfermagem, segundo YURA et al. ${ }^{12}$, liderança é um processo através do qual uma pessoa, que é o enfermeiro, influencia as ações de outros para a determinação e a consecução de objetivos, o que implica em definir e planejar a assistência de enfermagem num cenário interativo.

GALVÃO ${ }^{4}$ ressalta que é através da liderança "que o enfermeiro tenta conciliar os objetivos organizacionais com os objetivos do grupo da enfermagem, buscando o aprimoramento da prática profissional e principalmente o alcance de uma assistência de enfermagem adequada".

A amplitude e complexidade da temática liderança, aliada à escassez de estudos na enfermagem brasileira, principalmente na área de enfermagem cirúrgica, são fatores que têm determinado a necessidade de pesquisas.

Procurando oferecer nossa contribuição para o estudo da temática em questão, a presente investigação orientou-se para o enfermeiro de unidade de internação cirúrgica, considerando que compete ao enfermeiro deste setor a administração da assistência ao paciente cirúrgico, conforme análise realizada por TREVIZAN ${ }^{10}$. A autora destaca o reconhecimento da função administrativa centrada na assistência ao paciente como sendo a essência do trabalho do enfermeiro no contexto hospitalar. E nós ressaltamos a relevância da liderança nesse cenário.

Salientamos ainda que, de acordo com FONTES et al. ${ }^{3}$, o paciente frente a perspectiva da cirurgia fica "assediado pelo medo, medo do desconhecido, da morte, da anestesia ou da possibilidade de um câncer, que pode

\footnotetext{
* Enfermeira. Professor Doutor junto ao Departamento de Enfermagem Geral e Especializada da Escola de Enfermagem de Ribeirão Preto da Universidade de São Paulo

** Enfermeira. Professor Titular junto ao Departamento de Enfermagem Geral e Especializada da Escola de Enfermagem de Ribeirão Preto da Universidade de São Paulo
} 
ser aparente de imediato ou não; mas há outros medos mais intangíveis e latentes, aqueles por exemplo relativos à perda do emprego, à necessidade de sustentar a família ou à possibilidade de deficiência física permanente". Ao nosso ver, devido as características específicas de todo paciente e em especial do paciente cirúrgico, a assistência de enfermagem individualizada e qualificada é de suma importância e, vários estudos corroboram com nossa afirmação, como os de PANZA ${ }^{7}$; FERRAZ \& SALZANO $^{2}$ e RODRIGUES ${ }^{8}$. Desta forma, concordamos com TREVIZAN et al. ${ }^{11}$, quando afirmam que a função administrativa do enfermeiro deve privilegiar a assistência ao paciente e ser norteada "pela compreensão e pelo conhecimento do paciente como pessoa e de suas necessidades específicas. Este conhecimento orientará as ações do enfermeiro, no sentido de fazer implementar a assistência de enfermagem que os pacientes necessitam. Para tanto, ele deverá adequar princípios e medidas administrativas para a decisão e solução de problemas específicos e para a administração de seu pessoal".

Partindo das considerações expostas acima, acreditamos que o enfermeiro de unidade de internação cirúrgica necessita desenvolver e utilizar a habilidade de liderança, a qual consiste em um recurso fundamental para todo administrador.

Para fundamentarmos o presente estudo buscamos um modelo de liderança que pudesse ser aplicado no contexto organizacional-hospitalar. O modelo selecionado foi o de HERSEY \& BLANCHARD ${ }^{5}$, denominado Liderança Situacional. A seguir, apresentamos os conceitos-chave deste modelo.

\section{FUNDAMENTAÇÃO TEÓRICA}

HERSEY \& BLANCHARD ${ }^{5}$ definem liderança como "o processo de influenciar as atividades de indivíduos ou grupos para a consecução de um objetivo numa dada situação". O estilo de liderança de uma pessoa é definido pelos autores como sendo "o padrão comportamental que ela manifesta quando procura influenciar as atividades de outras, como é visto por essas outras pessoas". Esse conceito envolve uma combinação de dois tipos de comportamentos, tarefa e relacionamento. Acrescentam ainda, que o comportamento de tarefa "é a medida com que o líder dirige as pessoas, dizendo-lhes o que fazer, quando fazê-lo, onde e como fazê-lo. Significa estabelecer-lhes objetivos e definir os seus papéis". O comportamento de relacionamento, por sua vez, "é a medida com que um líder se empenha em comunicar-se bilateralmente com as pessoas, dando-lhes apoio, encorajamento e "carícias psicológicas". Significa ouvir ativamente as pessoas e apoiar-lhes os esforços".

Outro conceito fundamental da Liderança Situacional refere-se à maturidade dos liderados. Para HERSEY \& BLANCHARD ${ }^{5}$ a maturidade é definida como "a capacidade e a disposição das pessoas em assumir a responsabilidade de dirigir seu próprio comportamento". Os autores incluem neste conceito de maturidade duas dimensões: maturidade de trabalho (capacidade) e maturidade psicológica (disposição).

A primeira dimensão (maturidade de trabalho) refere-se a capacidade de fazer alguma coisa, está relacionada com o conhecimento e a capacidade técnica. A segunda dimensão (maturidade psicológica) está relacionada com a disposição ou motivação para realizar alguma coisa. Refere-se à confiança em si mesmo e ao empenho.

Salientamos que as dimensões do conceito de maturidade (capacidade e disposição) devem ser consideradas somente em relação a uma tarefa específica a ser realizada; não podemos dizer que um liderado ou grupo é maduro ou imaturo num sentido total.

A partir dos conceitos acima descritos podemos compreender a definição deste modelo proposto pelos autores. A Liderança Situacional "baseia-se numa interrelação entre a quantidade de orientação e direção (comportamento de tarefa) que o líder oferece, a quantidade de apoio sócio-emocional (comportamento de relacionamento) dado pelo líder e o nível prontidão ("maturidade") dos subordinados no desempenho de uma tarefa, função ou objetivo específico". . Na Figura 1 visualizamos o esquema geral desse modelo.

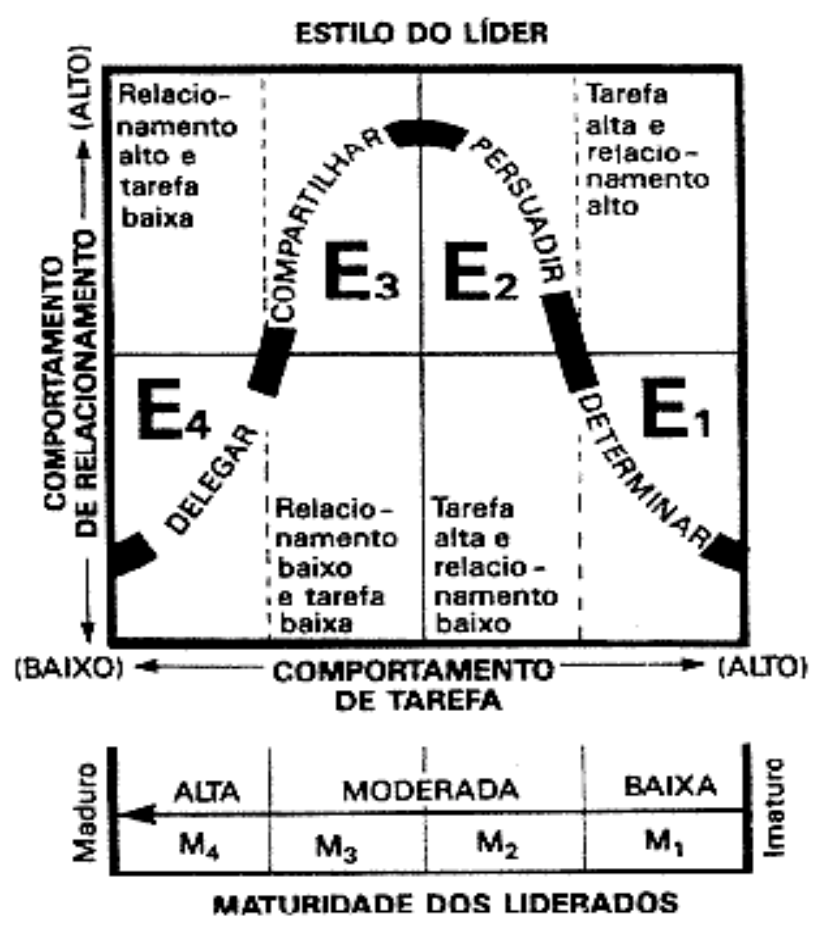

Figura 1 - Esquema geral da liderança situacional

Fonte: HERSEY,P.; BLANCHARD,K.H. Psicologia para administradores: a teoria e as técnicas da liderança situacional. Trad. Edwino A. Royer. São Paulo: Editora Pedagógica e Universitária, 1986. p.189. 
Conforme podemos observar na Figura 1, os autores da Liderança Situacional propõem quatro estilos de liderança: determinar $\left(\mathrm{E}_{1}\right)$, persuadir $\left(\mathrm{E}_{2}\right)$, compartilhar $\left(E_{3}\right)$ e delegar $\left(E_{4}\right)$, os quais são uma combinação de comportamento de tarefa e de relacionamento.

A maturidade dos liderados é apresentada dentro de um contínuo (imaturo/maduro) em quatro níveis: baixo $\left(\mathrm{M}_{1}\right)$, baixo a moderado $\left(\mathrm{M}_{2}\right)$, moderado a alto $\left(\mathrm{M}_{3}\right)$ e alto $\left(\mathrm{M}_{4}\right)$.

Desta forma, o conceito básico da Liderança Situacional é que "não existe um único modo melhor de influenciar as pessoas. $\mathrm{O}$ estilo de liderança que uma pessoa deve adotar com indivíduos ou grupos depende do nível de maturidade das pessoas que o líder deseja influenciar 5 ".

Passaremos a realizar uma síntese dos quatro estilos de liderança propostos pelos autores.

$\mathrm{O}$ estilo de liderança determinar $\left(\mathbf{E}_{\mathbf{I}}\right)$ é apropriado para pessoas com nível de maturidade baixo $\left(\mathrm{M}_{\mathrm{I}}\right)$; geralmente essas pessoas não possuem capacidade e nem disposição para assumirem responsabilidade de fazer algo. O líder assume um estilo diretivo, onde a orientação é clara, específica e a supervisão rigorosa; define as atividades que as pessoas devem fazer, como, quando e onde devem executá-las. Este estilo envolve um comportamento alto de tarefa e baixo de relacionamento.

Para pessoas que não tem capacidade, mas sentem disposição em assumir a responsabilidade de uma determinada tarefa, ou seja, com nível de maturidade entre baixo a moderado $\left(\mathrm{M}_{2}\right)$, o estilo de liderança correspondente é o persuadir $\left(\mathbf{E}_{\mathbf{2}}\right)$. Neste estilo apesar do líder assumir um comportamento diretivo, ele procura ao mesmo tempo apoiar e reforçar a disposição do liderado; através de explicações e comunicação bilateral ele tenta convencer o liderado a adotar o comportamento desejado. Freqüentemente o liderado segue adiante se ele compreender a razão da decisão tomada pelo líder e se o mesmo oferecer algum auxílio e direção. Este estilo implica um comportamento alto tanto para tarefa como para relacionamento.

As pessoas com nível de maturidade entre moderado a alto $\left(\mathrm{M}_{3}\right)$ têm capacidade, entretanto, não possuem disposição para realizar o que o líder deseja. Assim, o líder precisa apoiar os esforços do liderado, para ele poder utilizar a capacidade que já possui. O estilo compartilhar $\left(\mathbf{E}_{3}\right)$ é o mais apropriado para este nível de maturidade, é um estilo participativo, de apoio e não diretivo, ou seja, líder e liderado participam juntos do processo de tomada de decisão. Esse estilo envolve um comportamento alto de relacionamento e baixo de tarefa.

Para liderado que tem capacidade e disposição para assumir responsabilidades, ou seja, nível de maturidade alto $\left(\mathrm{M}_{4}\right)$, o estilo de liderança delegar $\left(\mathbf{E}_{\mathbf{4}}\right)$ é o mais eficaz. O líder pode ainda identificar o problema mas, cabe ao liderado a responsabilidade de desenvolver alternativas para solucioná-lo; ele decide como, quando e onde fazer as coisas. Esse estilo implica um comportamento baixo tanto para tarefa como para relacionamento.

De acordo com HERSEY \& DULT ${ }^{6}$, a chave para aplicação da Liderança Situacional consiste em diagnosticar o nível de maturidade do liderado e o líder adotar o estilo de liderança apropriado frente a uma determinada tarefa.

Após a apresentação dos conceitos-chave da Liderança Situacional, destacamos ainda, que a ênfase deste modelo recai sobre o comportamento do líder em relação aos liderados frente a uma situação específica. Assim, este modelo consiste no referencial teórico selecionado para embasarmos o presente estudo. $\mathrm{Na}$ sequiência, apresentamos o objetivo e o procedimento metodológico.

\section{OBJETIVO}

A presente investigação tem como objetivo: - identificar e analisar a correspondência de opinião entre o enfermeiro e um elemento do pessoal auxiliar de enfermagem sobre o estilo de liderança exercido pelo enfermeiro de unidade de internação cirúrgica, em relação às seis categorias de atividades assistenciais desenvolvidas na unidade.

\section{PROCEDIMENTO METODOLÓGICO}

\subsection{Primeira Fase: Construção e Validação dos Instrumentos}

A elaboração dos instrumentos utilizados nesta investigação foi realizada em três etapas: 1) construção dos instrumentos; 2) validação aparente e de conteúdo; 3 ) testagem dos instrumentos.

$\mathrm{Na}$ primeira etapa foram construídos dois instrumentos (Integração de Maturidade e Estilo/avaliação do líder e Integração de Maturidade e Estilo/avaliação do liderado). A construção destes instrumentos, foi baseada nos estudos de HERSEY \& BLANCHARD ${ }^{5}$, na literatura e na nossa vivência profissional.

Após esta etapa realizamos a validação aparente e de conteúdo. Solicitamos a cinco juizes que analisassem os instrumentos. Os juizes eram docentes da Escola de Enfermagem de Ribeirão Preto, os quais tinham experiência com a temática liderança e/ou validação de instrumento. Esses juizes sugeriram várias alterações, as 
quais aceitamos na sua maioria.

Realizamos um teste piloto em uma unidade de internação cirúrgica de um hospital governamental. Cinco enfermeiros preencheram o instrumento Integração de Maturidade e Estilo (avaliação do líder) e cinco elementos do pessoal auxiliar (técnicos e auxiliares de enfermagem) responderam o instrumento Integração de Maturidade e Estilo (avaliação do liderado). Esses sujeitos levantaram algumas dúvidas, que acarretaram novas modificações. Após esta etapa, elaboramos a versão final dos instrumentos que passaremos a descrever.

$\mathrm{O}$ instrumento Integração de Maturidade e Estilo $^{* * *}$ (avaliação do líder) contém duas partes. Na primeira parte, constam alguns dados gerais de identificação, a segunda refere-se ao estilo de liderança que o enfermeiro está usando com um de seus liderados, frente a uma categoria de atividades assistenciais desenvolvidas na unidade de internação cirúrgica.

$\mathrm{O}$ instrumento Integração de Maturidade e Estilo (avaliação do liderado) consta também de duas partes; a primeira relativa a alguns dados gerais de identificação, na segunda o liderado determina o estilo de liderança que o enfermeiro está usando com ele, frente a uma categoria de atividades assistenciais desenvolvidas na unidade de internação cirúrgica.

As atividades assistenciais (atividades relativas ao cuidado direto de enfermagem, proporcionadas ao paciente cirúrgico adulto) foram agrupadas em categorias a partir da literatura e da vivência profissional enquanto enfermeira e docente na área de enfermagem cirúrgica, da seguinte forma:

1. Categoria cuidados básicos - compreende os cuidados relativos à verificação de sinais vitais e higiene corporal;

2. Categoria administração de medicamentos compreende os cuidados relativos à administração de medicação por via oral, intramuscular, intradérmica, subcutânea e endovenosa;

3. Categoria procedimentos invasivos - compreende os cuidados relativos à sondagem vesical, nasogástrica e retal;

4. Categoria preparo do paciente cirúrgico no préoperatório - compreende os cuidados relativos ao jejum, tricotomia e coleta de exames;

5. Categoria assistência ao paciente cirúrgico no encaminhamento para o Centro Cirúrgico compreende os cuidados relativos ao transporte, retirada de prótese, vestimenta e documentação;

6. Categoria assistência ao paciente cirúrgico no pós-operatório - compreende os cuidados relativos ao controle de entrada e saída de líquidos (cuidados com soros, sondas e drenos).

\subsection{Segunda Fase: Aplicação dos Instrumentos}

O trabalho foi desenvolvido em dois hospitais gerais da cidade de Ribeirão Preto - São Paulo, sendo um hospital governamental (hospital A) e o outro, instituição particular (hospital B). A amostra consistiu dos enfermeiros e de elementos do pessoal auxiliar (técnicos e auxiliares de enfermagem) que atuavam na unidade de internação cirúrgica dos hospitais selecionados, na ocasião da coleta de dados (a coleta de dados ocorreu nos meses de março e abril de 1994).

A coleta de dados foi realizada através da aplicação do instrumento Integração de Maturidade e Estilo (avaliação do líder) nos enfermeiros e do instrumento Integração de Maturidade e Estilo (avaliação do liderado) no pessoal auxiliar.

No hospital A, participaram do estudo treze enfermeiros, dois técnicos e onze auxiliares de enfermagem. Em relação ao hospital B, também participaram desta investigação treze enfermeiros, quatro técnicos e nove auxiliares de enfermagem. Assim, a amostra da presente pesquisa consistiu de cinqüenta e dois sujeitos.

Ressaltamos ainda, que a amostra dos sujeitos foi pareada, ou seja, ao responder o instrumento o enfermeiro $X$ teve como referência o técnico de enfermagem $Z$ e vice-versa.

\section{RESULTADOS E DISCUSSÃO}

\subsection{Caracterização da amostra}

Dos enfermeiros investigados no hospital A, onze $(84,6 \%)$ eram do sexo feminino e dois $(15,3 \%)$ do sexo masculino; oito $(61,5 \%)$ encontravam-se na faixa etária de trinta e um a trinta e cinco anos e três $(23 \%)$ na faixa etária de trinta e seis a quarenta anos, um $(7,6 \%)$ na faixa etária de vinte e cinco a trinta anos e também apenas um (7,6\%) na faixa etária de quarenta e um a quarenta e cinco anos. Em relação ao tempo de atuação, seis $(46,1 \%)$ enfermeiros trabalhavam na unidade de seis a dez anos, quatro $(30,7 \%)$ de um a cinco anos e três $(23 \%)$ de onze a quinze anos.

Do pessoal auxiliar estudado, nove elementos $(69,2 \%)$ eram do sexo feminino e quatro $(30,7 \%)$ do sexo masculino. Em relação a categoria profissional onze

\footnotetext{
* Os instrumentos utilizados nesta pesquisa podem ser encontrados na íntegra na seguinte bibliografia:

GALVÃO, C.M. Liderança Situacional: uma contribuição ao trabalho do enfermeiro-líder no contexto hospitalar. Ribeirão Preto, 1995. 117p. Tese (Doutorado) - Escola de Enfernagem de Ribeirão Preto, Universidade de São Paulo.
} 
$(84,6 \%)$ eram auxiliares de enfermagem e dois $(15,3 \%)$ eram técnicos de enfermagem. Destes sujeitos, cinco $(38,4 \%)$ encontravam-se na faixa etária de trinta e seis a quarenta anos, quatro $(30,7 \%)$ na faixa etária de quarenta e um a quarenta e cinco anos, dois $(15,3 \%)$ na faixa etária de trinta e um a trinta e cinco anos. Segundo o tempo de atuação, do pessoal auxiliar investigado, quatro elementos $(30,7 \%)$ trabalhavam na unidade de seis a dez anos, três $(23 \%)$ de onze a quinze anos, três $(23 \%)$ de dezesseis a vinte anos, dois $(15,3 \%)$ de um a cinco anos e um $(7,6 \%)$ de vinte e um a vinte e cinco anos.

No hospital B, dos enfermeiros participantes, onze $(84,6 \%)$ eram do sexo feminino e dois $(15,3 \%)$ do sexo masculino. Destes profissionais, dez $(76,9 \%)$ encontravam-se na faixa etária de vinte e cinco a trinta anos, dois $(15,3 \%)$ na faixa etária de trinta e seis a quarenta anos e um $(7,6 \%)$ na faixa etária de trinta e um a trinta e cinco anos. Em relação ao tempo de atuação, doze $(92,3 \%)$ enfermeiros trabalham na unidade de um a cinco anos, um $(7,6 \%)$ de seis a dez anos.

Em relação, ao pessoal auxiliar, doze elementos $(92,3 \%)$ eram do sexo feminino e um $(7,6 \%)$ do sexo masculino; nove $(69,2 \%)$ eram auxiliares de enfermagem e quatro $(30,7 \%)$ eram técnicos de enfermagem. Destes sujeitos, seis $(46,1 \%)$ encontravam-se na faixa etária de vinte e cinco a trinta anos, quatro $(30,7 \%)$ na faixa etária de trinta e seis a quarenta anos, dois $(15,3 \%)$ na faixa etária de trinta e um a trinta e cinco anos e apenas um $(7,6 \%)$ na faixa etária de quarenta e um a quarenta e cinco anos. Ainda, em relação ao tempo de atuação, onze elementos $(84,6 \%)$ trabalhavam na unidade de internação cirúrgica de um a cinco anos, dois $(15,3 \%)$ de seis a dez anos.

\subsection{O estilo de liderança exercido pelo enfermeiro de unidade de internação cirúrgica}

Na primeira parte dos instrumentos encontramos dados gerais sobre os sujeitos investigados, os quais já descrevemos no item anterior. $\mathrm{Na}$ segunda parte do instrumento Integração de Maturidade e Estilo (avaliação do líder) os enfermeiros responderam o estilo de liderança utilizado com o liderado em relação a uma categoria de atividades assistenciais desenvolvidas na unidade de internação cirúrgica. O pessoal auxiliar ao responder também a segunda parte do instrumento Integração de Maturidade e Estilo (avaliação do liderado) determinou o estilo de liderança que o enfermeiro usou com ele, frente a uma categoria de atividades assistenciais desenvolvidas na unidade de internação cirúrgica.

Nos instrumentos aplicados, abaixo de cada categoria de atividades assistenciais existem quatro descrições, as quais retratam estilos de liderança. A descrição "dá instruções específicas e supervisiona estreitamente o desempenho" refere-se ao estilo determinar $\left(\mathrm{E}_{1}\right)$; “explica suas decisões e dá oportunidade de esclarecimento" relaciona-se com o estilo persuadir (E2); "compartilha idéias e o processo decisório" ao estilo compartilhar (E3) e "concede a responsabilidade pelas decisões e pela implantação" ao estilo delegar (E4). A seguir, passaremos a discorrer sobre os achados encontrados nos hospitais A (Quadro 1) e B (Quadro 2).

A categoria cuidados básicos (1) compreendia os procedimentos relativos à verificação de sinais vitais e higiene corporal. Conforme podemos observar nos quadros 1 e 2 assinalamos com asteriscos (*) os pares de sujeitos que obtiveram correspondência entre si, ou seja, líder e liderado possuíam a mesma opinião em relação ao estilo de liderança do enfermeiro nesta categoria.

Quadro 1 - Distribuição dos estilos de liderança dos enfermeiros $\left(E_{1}-E_{4}\right)$, segundo os sujeitos participantes, nas categorias de atividades assistenciais (1-6) no hospital A.

\begin{tabular}{|c|c|c|c|c|c|c|}
\hline \multirow[b]{2}{*}{ SUJEITOS } & \multicolumn{6}{|c|}{ ATTVDADES ASSISTENCLIS } \\
\hline & $\mathbf{1}$ & 2 & 3 & 4 & 5 & 6 \\
\hline $\begin{array}{l}\mathrm{ENF}_{2} \\
\text { PAX, }\end{array}$ & $\begin{array}{l}\mathrm{E} \\
\mathrm{E}\end{array}$ & $\underset{\mathrm{E}}{\mathrm{E}}$ & $\frac{\mathrm{E}_{2}}{\mathrm{E}}$ & $\stackrel{\mathrm{E}}{\mathrm{E}}$ & $\frac{\mathrm{E}}{\mathrm{E}}$ & 至 \\
\hline $\begin{array}{l}\mathrm{ENF}_{2} \\
\text { PAK }\end{array}$ & $\underset{\mathrm{E}_{1}}{\mathrm{E}}$ & $\underset{+5}{E}$ & $\frac{\mathrm{E}_{\mathrm{H}}}{\mathrm{E}_{1}}$ & $\underset{\mathrm{E}_{1}}{\mathrm{E}_{2}}$ & $\frac{\mathrm{E}_{2}}{\mathrm{E}_{1}}$ & $\underset{\mathrm{E}}{\mathrm{E}}$ \\
\hline $\begin{array}{l}\mathrm{ENF}_{2} \\
\mathrm{PAX}\end{array}$ & 至 & $\frac{\mathrm{E}_{\mathrm{F}}}{\mathrm{E}}$ & $\frac{\mathrm{E}}{\mathrm{E}}$ & $\underset{\mathrm{E}}{\mathrm{E}}$ & $\underset{\mathrm{E}}{\mathrm{E}}$ & $\underset{\mathrm{E}}{\mathrm{E}}$ \\
\hline $\begin{array}{l}\text { ENF, } \\
\text { PAX, }\end{array}$ & $\underset{+E_{1}}{E}$ & $\underset{\mathrm{E}}{\mathrm{E}}$ & $\underset{\mathrm{E}}{\mathrm{E}}$ & 点 & $\frac{\mathrm{E}}{\mathrm{E}}$ & $\underset{\mathrm{E}}{\mathrm{E}}$ \\
\hline $\begin{array}{l}\mathrm{ENF} \\
\text { PAX }\end{array}$ & $\frac{\mathrm{E}}{\mathrm{E}}$ & $\frac{\mathrm{E}_{\mathrm{F}}}{\mathrm{E}}$ & ${ }_{E_{1}}$ & $\underset{\mathrm{E}}{\mathrm{E}}$ & $\mathrm{WE}_{\mathrm{E}}$ & ${ }^{\mathrm{E}}$ \\
\hline $\begin{array}{l}\mathrm{ENF} \\
\text { PAX }\end{array}$ & $\frac{\mathrm{E}}{\mathrm{E}}$ & 豆 & $\frac{\mathrm{E}_{2}}{\mathrm{E}}$ & $\underset{+E_{1}}{E}$ & $\frac{\mathrm{E}}{\mathrm{E}}$ & $\underset{\mathrm{E}_{1}}{\mathrm{E}}$ \\
\hline $\begin{array}{l}\mathrm{ENF}_{\mathrm{I}} \\
\mathrm{PAX}\end{array}$ & $\frac{\mathrm{E}}{\mathrm{E}_{1}}$ & $\underset{\mathrm{E}}{\mathrm{E}}$ & $\begin{array}{l}\mathrm{E} \\
\mathrm{E}_{1}\end{array}$ & $\underset{\mathrm{E}}{\mathrm{E}}$ & $\frac{\mathrm{E}_{2}}{\mathrm{E}_{1}}$ & $\underset{\mathrm{E}}{\mathrm{E}}$ \\
\hline $\begin{array}{l}\mathrm{ENF} \\
\text { PAX }\end{array}$ & $\underset{\mathrm{E}}{\mathrm{E}}$ & $\underset{\mathrm{E}}{\mathrm{E}}$ & $\underset{\mathrm{E}}{\mathrm{E}}$ & $\underset{\mathrm{E}}{\mathrm{E}}$ & 啶 & $\underset{\mathrm{E}}{\mathrm{E}}$ \\
\hline $\begin{array}{l}\mathrm{ENF} \\
\text { PAX }\end{array}$ & $\frac{\mathrm{E}_{2}}{\mathrm{E}_{1}}$ & $\underset{\mathrm{E}_{1}}{\mathrm{E}}$ & $\underset{E_{1}}{E}$ & 佂 & $\frac{\mathrm{E}}{\mathrm{E}_{1}}$ & $\begin{array}{l}\mathrm{E} \\
\mathrm{E}_{1}\end{array}$ \\
\hline $\begin{array}{l}\mathrm{EHF} \\
\mathrm{PAX}_{\mathrm{D}}\end{array}$ & $\frac{\mathrm{E}_{2}}{\mathrm{E}_{1}}$ & $\frac{\mathrm{E}_{2}}{\mathrm{E}_{1}}$ & $\underset{\mathrm{E}}{\mathrm{E}}$ & $\underset{+}{E}$ & $\frac{\mathrm{E}}{\mathrm{E}}$ & $\underset{H}{E}$ \\
\hline $\begin{array}{l}\mathrm{EHF}_{11} \\
\operatorname{PAX}_{11}\end{array}$ & $\frac{\mathrm{E}_{1}}{\mathrm{E}_{1}}$ & $\underset{E_{1}}{E_{1}}$ & $\begin{array}{l}\frac{E_{1}}{E_{1}} \\
\end{array}$ & $\underset{\mathrm{E}}{\mathrm{E}}$ & $\underset{\mathrm{E}}{\mathrm{E}}$ & $\underset{\mathrm{E}_{1}}{\mathrm{E}}$ \\
\hline $\begin{array}{l}E N F_{a} \\
\operatorname{PAx} x_{n}\end{array}$ & $\underset{H}{E}$ & $\frac{\mathrm{E}_{1}}{\mathrm{E}_{1}}$ & $\begin{array}{l}\frac{\mathrm{E}_{1}}{\mathrm{E}_{1}} \\
\end{array}$ & $\frac{\mathrm{E}_{1}}{\mathrm{E}_{4}}$ & $\begin{array}{l}\mathrm{E} \\
\mathrm{E}\end{array}$ & $\begin{array}{l}\mathrm{E} \\
\mathrm{E}\end{array}$ \\
\hline $\begin{array}{l}\mathrm{EHF}_{\mathrm{n}} \\
\text { PAX }_{n}\end{array}$ & $\begin{array}{l}\underset{\mathrm{E}}{\mathrm{E}} \\
\mathrm{E}\end{array}$ & $\begin{array}{l}\underset{\mathrm{E}}{\mathrm{E}} \\
\mathrm{F}\end{array}$ & $\begin{array}{l}\frac{\mathrm{E}_{2}}{\mathrm{E}} \\
\mathrm{F}\end{array}$ & $\begin{array}{l}\mathrm{E} \\
\mathrm{E}\end{array}$ & $\begin{array}{l}\mathrm{E}_{1} \\
\mathrm{E}\end{array}$ & $\begin{array}{l}\mathrm{E} \\
\mathrm{E}\end{array}$ \\
\hline
\end{tabular}

$\mathrm{ENF}=$ Enfermeiro $\quad \mathrm{E}_{1}=$ Determinar $\quad \mathrm{E}_{3}=$ Compartilhar $\mathrm{PAX}=$ Pessoal Auxiliar $\quad \mathrm{E}_{2}=$ Persuadir $\quad \mathrm{E}_{4}=$ Delegar 
Quadro 2 - Distribuição dos estilos de liderança dos enfermeiros $\left(E_{1} . E_{4}\right)$, segundo os sujeitos participantes, nas categorias de atividades assistenciais (1-6) no hospital $B$.

\begin{tabular}{|c|c|c|c|c|c|c|}
\hline \multirow[b]{2}{*}{ SUJETTOS } & \multicolumn{6}{|c|}{ ATIVIDADES ASSISTENCLAIS } \\
\hline & $\mathbf{l}$ & 2 & 3 & 4 & 5 & 6 \\
\hline$\frac{\mathrm{ENF}}{\mathrm{PAX}_{4}}$ & $\frac{\mathrm{E}_{2}}{\mathrm{E}}$ & $\frac{\mathrm{F}_{2}}{\mathrm{E}}$ & $\frac{\mathrm{F}_{1}}{\mathrm{E}_{2}}$ & $\frac{\mathrm{F}_{1}}{\mathrm{E}_{2}}$ & $\underset{\text { 悎 }}{\mathrm{E}}$ & $\frac{\mathrm{E}}{\mathrm{E}}$ \\
\hline$\frac{\mathrm{ENF}_{2}}{\mathrm{PAB}_{2}}$ & $\frac{\mathrm{E}}{\mathrm{F}}$ & $\frac{\mathrm{F}_{2}}{\mathrm{~F}}$ & $\frac{\mathrm{E}}{\mathrm{E}}$ & $\frac{\mathrm{F}_{2}}{\mathrm{~F}}$ & 点 & 㝕 \\
\hline $\begin{array}{l}\mathrm{ENF}_{2} \\
\mathrm{PAX}_{1}\end{array}$ & $\begin{array}{l}\mathrm{E}_{\mathrm{F}} \\
\mathrm{E}\end{array}$ & $\frac{\mathrm{E}_{2}}{\mathrm{E}}$ & $\frac{\mathrm{F}}{\mathrm{F}}$ & $\frac{\mathrm{E}}{\mathrm{E}}$ & $\frac{\mathrm{E}}{\mathrm{E}}$ & $\stackrel{\mathrm{E}}{\mathrm{E}}$ \\
\hline$\frac{\mathrm{DNF}}{\mathrm{PAX}}$ & $\mathrm{F}$ & $\frac{\mathrm{E}}{\mathrm{E}}$ & $\frac{\mathrm{E}}{\mathrm{E}}$ & $\frac{\mathrm{E}}{\mathrm{E}}$ & $\frac{\mathrm{E}}{\mathrm{E}}$ & $\frac{\mathrm{E}}{\mathrm{E}}$ \\
\hline $\begin{array}{l}\mathrm{ENF} \\
\text { PAX }\end{array}$ & $\frac{\mathrm{E}}{\mathrm{E}}$ & $\frac{\mathrm{F}_{2}}{\mathrm{E}}$ & $\frac{\mathrm{E}}{\mathrm{E}}$ & $\frac{\mathrm{F}_{2}}{\mathrm{E}}$ & $\frac{\mathrm{E}}{\mathrm{E}}$ & $\frac{\mathrm{E}}{\mathrm{E}}$ \\
\hline$\frac{\mathrm{ENF}}{\text { PAK }}$ & $\frac{\mathrm{E}}{\mathrm{E}}$ & $\frac{\mathrm{E}}{\mathrm{E}}$ & $\frac{\mathrm{E}}{\mathrm{E}}$ & $\frac{\mathrm{F}}{\mathrm{E}}$ & $\frac{\mathrm{E}}{\mathrm{E}}$ & $\mathrm{E}$ \\
\hline$\frac{\mathrm{BNF}_{\mathrm{N}}}{\mathrm{PHAX}_{\mathrm{T}}}$ & 晖 & 萤 & $\frac{\mathrm{E}_{2}}{\mathrm{E}}$ & $\frac{\mathrm{E}_{1}}{\mathrm{E}_{1}}$ & 点 & F \\
\hline$\frac{\mathrm{DNF}}{\mathrm{PAR}}$ & $\frac{\mathrm{E}}{\mathrm{E}}$ & E & E & $\frac{\mathrm{E}}{\mathrm{E}}$ & $\frac{\mathrm{E}}{\mathrm{E}}$ & $\frac{\mathrm{E}}{\mathrm{E}}$ \\
\hline $\begin{array}{l}\mathrm{ENF} \\
\mathrm{PAX}\end{array}$ & $\frac{\mathrm{E}}{\mathrm{E}}$ & $\frac{\mathrm{E}}{\mathrm{E}}$ & $\underset{n}{\mathrm{E}}$ & $\frac{\mathrm{F}_{2}}{\mathrm{E}}$ & 卧 & $\underset{ }{E}$ \\
\hline $\begin{array}{l}\mathrm{EHF}, \\
{ }_{\text {PAX }}\end{array}$ & $\frac{\mathrm{F}_{\mathrm{F}}}{\mathrm{E}_{1}}$ & $\frac{E_{2}}{E_{1}}$ & $\frac{\mathrm{E}}{\mathrm{E}}$ & $\begin{array}{l}\mathrm{E}_{1} \\
\mathrm{E}_{1}\end{array}$ & $\begin{array}{l}\mathrm{E}_{2} \\
\mathrm{E}_{1}\end{array}$ & $\frac{\mathrm{E}}{\mathrm{E}}$ \\
\hline $\begin{array}{l}\mathrm{EFFF}_{\mu} \\
\text { PAX }_{41}\end{array}$ & 豆 & $\frac{\mathrm{F}_{2}}{\mathrm{E}}$ & $\frac{\mathrm{E}}{\mathrm{F}}$ & $\frac{\mathrm{F}_{2}}{\mathrm{E}}$ & $\frac{\mathrm{E}}{\mathrm{E}}$ & 売 \\
\hline $\begin{array}{l}\mathrm{ENF}_{\mathrm{a}} \\
\mathrm{PARx}_{\mathrm{a}}\end{array}$ & 婜 & $\frac{\mathrm{F}_{2}}{\mathrm{E}_{\mathrm{L}}}$ & $\begin{array}{l}E_{2} \\
E_{1}\end{array}$ & $\begin{array}{l}\mathrm{E} \\
\mathrm{E}\end{array}$ & $\begin{array}{l}\mathrm{E} \\
\mathrm{E}\end{array}$ & $\frac{\mathrm{E}_{2}}{\mathrm{E}_{\mathrm{i}}}$ \\
\hline $\begin{array}{l}\mathrm{ENF} F_{n} \\
\mathrm{PACx}_{n}\end{array}$ & $\underset{E_{1}}{\mathrm{E}}$ & $\underset{\mathrm{E}}{\mathrm{E}}$ & $\begin{array}{l}\mathrm{E}_{\mathrm{G}} \\
\mathrm{E}\end{array}$ & 志 & $\begin{array}{l}\mathrm{F}_{2} \\
\mathrm{~F}_{1}\end{array}$ & $\frac{\mathrm{F}_{2}}{\mathrm{E}_{\mathrm{i}}}$ \\
\hline
\end{tabular}

$\mathrm{ENF}=$ Enfermeiro $\quad \mathrm{E}_{1}=$ Determinar $\quad \mathrm{E}_{3}=$ Compartilhar $\mathrm{PAX}=$ Pessoal Auxiliar $\quad \mathrm{E}_{2}=$ Persuadir $\quad \mathrm{E}_{4}=$ Delegar

$\mathrm{Na}$ categoria em questão, nos hospitais A e B, foi o estilo de liderança $E_{3}$ (compartilhar) o que obteve maior índice de correspondência (quatro pares de sujeitos nos dois hospitais), ou seja, enfermeiro e pessoal auxiliar possuíam a mesma opinião em relação ao estilo de liderança do enfermeiro frente aos cuidados relativos à verificação de sinais vitais e higiene corporal.

$\mathrm{O}$ estilo de liderança $\mathrm{E}_{3}$ envolve comportamento baixo para tarefa e alto para relacionamento por parte do líder. A comunicação neste estilo é do tipo duas vias, o comportamento de apoio do líder está aumentado. Salientamos que uma característica específica neste estilo é que a direção do que deve ser feito é dada pelo liderado e não mais pelo líder. Ao empregar este estilo, o enfermeiro encoraja o elemento da equipe de enfermagem a tomar decisões.

$\mathrm{Na}$ categoria administração de medicamentos (2), a qual estava relacionada com os cuidados referentes a administração por via oral, intramuscular, intradérmica, subcutânea e endovenosa de medicação, foi o estilo $E_{2}$ (persuadir) o que apresentou maior índice de correspondência no hospital A (três pares de sujeitos); enquanto no hospital B apenas um par de sujeitos demonstrou correspondência e o estilo apontado foi o $\mathrm{E}_{3}$ (compartilhar).

No estilo de liderança persuadir $\left(\mathrm{E}_{2}\right)$ a comunicação do líder também é do tipo duas vias, nesse estilo ele diminui o comportamento diretivo e aumenta o comportamento de apoio. Implica comportamento alto tanto para tarefa como para relacionamento.

O enfermeiro, através de comunicação adequada, expõe ao elemento da equipe de enfermagem a melhor maneira de executar determinada atividade tendo como referência o seu conhecimento científico e a experiência profissional; ele traça um plano de orientação e supervisão para convencer o elemento da equipe de enfermagem a desenvolver a atividade em questão.

A categoria procedimentos invasivos(3) referiase aos cuidados relativos à sondagem vesical, nasogástrica e retal. Nesta categoria no hospital A, os estilos de liderança $E_{1}$ (determinar), $E_{2}$ (persuadir) e $E_{3}$ (compartilhar) demonstraram o mesmo índice de correspondência (um par de sujeitos em cada estilo). No hospital B, apenas um par de sujeitos apresentou correspondência e o estilo foi o $E_{1}$.

Já foram abordados os estilos de liderança compartilhar $\left(\mathrm{E}_{3}\right)$ e persuadir $\left(\mathrm{E}_{2}\right)$. Quanto ao estilo de liderança determinar $\left(\mathrm{E}_{1}\right)$ a comunicação do líder é do tipo mão única e ele define o papel do liderado dizendo o que fazer, como fazer, onde e quando. Esse estilo envolve comportamento alto de tarefa e baixo de relacionamento. É particularmente aplicado com pessoas que iniciam sua prática profissional na enfermagem.

Geralmente essas pessoas não possuem capacidade e nem disposição em relação ao desempenho de determinada atividade da assistência de enfermagem ao paciente. Assim, o enfermeiro deverá traçar um plano de orientação que torne essa pessoa apta a desenvolver aquela atividade. Nesse caso, o enfermeiro utilizando o estilo de liderança determinar, provavelmente tem grande probabilidade de obter sucesso.

$\mathrm{Na}$ categoria preparo do paciente cirúrgico no pré-operatório(4), a qual compreendia os cuidados referentes ao jejum, tricotomia e coleta de exames e na categoria assistência ao paciente cirúrgico no pósoperatório(6), a qual referia-se aos cuidados relativos ao controle de entrada e saída de líquidos, ou seja, cuidados 
com soros, sondas e drenos o estilo de liderança $\mathrm{E}_{1}$ (determinar) foi o que obteve maior índice de correspondência, no hospital A (quatro pares de sujeitos em cada categoria). Em relação ao hospital B, nas mesmas categorias apenas um par de sujeitos em cada uma delas, apresentou correspondência e o estilo indicado também foi o $\mathrm{E}_{1}$.

No hospital A, na categoria assistência ao paciente cirúrgico no encaminhamento para o Centro Cirúrgico(5), a qual estava relacionada com os cuidados referentes ao transporte, retirada de prótese, vestimenta e documentação, dois pares de sujeitos demonstraram correspondência, um para o estilo $\mathrm{E}_{1}$ (determinar) e um para o $\mathrm{E}_{4}$ (delegar). No hospital $\mathrm{B}$, nesta categoria de atividades assistenciais quatro pares de sujeitos obtiveram correspondência, e os estilos de liderança apontados foram o $\mathrm{E}_{2}$ (persuadir) por dois pares e $\mathrm{o} \mathrm{E}_{3}$ (compartilhar) pelos outros.

No estilo de liderança delegar $\left(\mathrm{E}_{4}\right)$ a comunicação do líder é do tipo duas vias, esse estilo implica comportamento baixo tanto para tarefa como para relacionamento. Cabe ao enfermeiro identificar a atividade que deverá ser feita e ao elemento da equipe de enfermagem desempenhá-la. O enfermeiro somente é solicitado quando ocorre complicações.

Ao compararmos os achados encontrados no hospital A com os do hospital B podemos verificar que a quantidade de correspondência entre o enfermeiro e o pessoal auxiliar relativo ao estilo de liderança do enfermeiro frente as categorias de atividades assistenciais estudadas foi maior no hospital A.

\section{CONCLUSÃO}

Conforme afirmam HERSEY \& BLANCHARD ${ }^{5}$, liderança é um processo, desta forma, estilos diferentes são necessários. Assim, ao tomarmos o conjunto de correspondência de opinião entre o enfermeiro e o elemento do pessoal auxiliar, sobre o estilo de liderança daquele profissional em relação às seis categorias de atividades assistenciais desenvolvidas nas unidades de internação cirúrgica das instituições estudadas, nossos achados apontaram que no hospital A (quadro 1) houve predominância dos estilos de liderança determinar $\left(E_{1}\right)$ e persuadir ( $\mathrm{E}_{2}$ ); no hospital $\mathrm{B}$, como mencionamos, o índice de correspondência de opinião entre os sujeitos foi bem menor (ver quadro2), sendo vinculado ao estilo de liderança compartilhar (E3) e ao estilo determinar (E1). Considerando mais uma vez, a quantidade de correspondência de opinião entre os sujeitos nos dois hospitais, observamos que os enfermeiros têm se dedicado aos estilos de liderança mais diretivos, orientando, portanto, seu comportamento sobretudo para a tarefa, sugerindo- nos que o pessoal auxiliar tem apresentado nível de maturidade baixo e baixo a moderado, ou então, que os enfermeiros têm utilizado inadequadamente o estilo gerencial. Ao nosso ver, alguns determinantes destes resultados sejam oriundos da burocratização dos hospitais e dos serviços de enfermagem, que intensificam por força do próprio esquema burocrático, estilos gerenciais mais diretivos. Este ponto de vista encontra apoio em TREVIZAN $^{10}$ que ao abordar sobre a função administrativa do enfermeiro no contexto hospitalar, constatou que $74 \%$ desta função é de caráter burocrático indicando "uma tendência para a rotinização e impessoalidade e, portanto, vinculação mais acentuada a normas pré-estabelecidas", ocasionando desta forma, adoção de estilos de liderança mais rígidos e diretivos. No hospital B houve indicação de correspondência sobre o estilo de liderança compartilhar (E3) nas categorias de atividades assistenciais: cuidados básicos, administração de medicamentos e assistência ao paciente cirúrgico no encaminhamento para o Centro Cirúrgico, sugerindo-nos que o pessoal auxiliar apresenta nível de maturidade moderado a alto e que os enfermeiros têm orientado seu comportamento mais para o relacionamento do que para tarefa.

Questões como estas são fundamentais e precisam ser aprofundadas em nosso meio para que possamos utilizar mais plenamente o potencial de nossos recursos humanos e a liderança por parte do enfermeiro. Desta forma, é necessário que o enfermeiro, além de compreender o processo de liderança relativo a uma determinada situação, precisa também conhecer e conciliar o nível de maturidade dos liderados, com o estilo de liderança apropriado. Assim, acreditamos que a Liderança Situacional poderá fornecer contribuição fundamental para o desempenho do enfermeiro-líder e de modo especial no que tange à articulação entre maturidade do liderado e estilo de liderança.

Para isso concordamos com HERSEY \& BLANCHARD $^{5}$ quando dizem que o líder precisa ter habilidade tanto para diagnosticar o nível de maturidade do liderado, como também para modificar o seu estilo de liderança para ser congruente com as alterações que podem ocorrer no nível de maturidade do liderado, seja, o grau que o enfermeiro conseguir adaptar o seu estilo de liderança em relação ao nível de maturidade do elemento da equipe de enfermagem frente a uma determinada atividade, indicará o seu sucesso ou não como líder.

\section{CONSIDERAÇÕES FINAIS}

A presente investigação teve como propósito dar continuidade aos nossos estudos sobre liderança do enfermeiro no contexto hospitalar, especificamente do enfermeiro de unidade de internação cirúrgica. 
No contexto hospitalar, cabe ao enfermeiro-líder buscar mecanismos que proporcionem ao paciente uma assistência individualizada e com qualidade, e ao pessoal de enfermagem, desenvolvimento do seu potencial, bem como o atendimento das suas necessidades. Além disso, os objetivos organizacionais não podem ser menosprezados; pelo contrário, devem ser atingidos através de recursos humanos competentes e satisfeitos.
Nesse cenário, entendemos que a liderança do enfermeiro seja de suma importância e que o embasamento teórico, um eixo norteador desse processo.

Acreditamos que, a Liderança Situacional poderá ser utilizada como estrutura teórica no que tange a habilidade de liderar do enfermeiro com vistas ao aprimoramento da qualidade da assistência de enfermagem prestada ao paciente/cliente e do desenvolvimento do potencial do pessoal de enfermagem.

\section{THE LEADERSHIP STYLE ADOPTED BY NURSES IN SURGICAL WARD UNITIES FOCUSSING THE SITUATIONAL LEADERSHIP}

The present study was oriented to the leadership theme focussing nurses inside surgical ward unities. As a theoretical reference, the authors used the Situational Leadership Model proposed by Hersey and Blanchard. This study aimed at analysing the correspondence between the opinions of nurses and auxiliary personnel about the leadership style exerted by nurses in the surgical ward unit regarding the six categories of the assistance activity that were studied. Authors noticed that nurses, from the two studied hospitals, adopted the directive leadership styles (E2/selling or E1/telling) with the auxiliary personnel.

KEY WORDS: nurse, surgical ward unities, situational leadership

\section{EL ESTILO DE LIDERAZGO ADOPTADO POR EL ENFERMERO DE UNIDAD QUIRÚRGICA, BAJO EL ENFOQUE DEL LIDERAZGO SITUACIONAL}

La presente investigación se orientó para la temática del liderazgo enfocando el enfermero de unidad quirúrgica. Como referencial teórico utilizamos el modelo de liderazgo propuesto por Hersey y Blanchard, denominado Liderazgo Situacional. El objetivo de éste estudio fue analizar la correspondencia de opinión entre el enfermero y el personeal auxiliar de enfermería sobre el estilo de liderazgo adoptado por el enfermero de la unidad de hospitalización quirúrgica en relación a las seis categorías de actividades asistenciales estudiadas. Constatamos que los enfermeros, en los dos hospitales estudiados, adoptan con el personal auxiliar los estilos de liderazgo más directivo, o sea, E2(persuadir) o E1(determinar).

TÉRMINOS CLAVES: enfermero, unidad de hospitalización quirúrgica, liderazgo situacional

\section{REFERÊNCIAS BIBLIOGRÁFICAS}

01. CHIAVENATO, I. Introdução à teoria geral da administração. $4^{\mathrm{a}}$ ed. São Paulo: Makron Books, 1993.

02. FERRAZ, E. R.; SALZANO, S. D. T. O paciente cirúrgico: suas expectativas e opiniões quanto ao cuidado de enfermagem no período transoperatório. Rev. Bras. Enfermagem, Brasília, v. 35, n. 1, p. 48-59, 1982.

03. FOnTES, M. C. C.; AlmeidA, M. S.; CARVALHO, D. D. O trauma cirúrgico: importância da orientação pré-operatório. Rev. Bras. Enfermagem, Brasília, v. 33, n. 2, p. 194200, 1980.

04. GALVÃO, C. M. Liderança do enfermeiro de centro cirúrgico. Ribeirão Preto, 1990. 69 p. Dissertação (Mestrado) - Escola de Enfermagem de Ribeirão Preto, Universidade de São Paulo.
05. HERSEY, P.; BLANCHARD, K.H. Psicologia para administradores: a teoria e as técnicas da liderança situacional. Trad. Edwino A. Royer. São Paulo: Editora Pedagógica e Universitária, 1986.

06. HERSEY, P.; DULT, B. W. Situational leadership in nursing. California: Appleton \& Lange, 1989.

07. PANZA, A. M. M. Efeito da visita pré-operatória da enfermeira de centro cirúrgico sobre o estresse do paciente no pré-operatório, no dia da cirurgia e no pós-operatório. São Paulo, 1977. 75 p. Dissertação (Mestrado) - Escola de Enfermagem, Universidade de São Paulo.

08. RODRIGUES, A. I. O paciente no sistema centro cirúrgico: um estudo sobre percepções e opiniões de paciente em relação ao período transoperatório. São Paulo, 1979. 147 p. Dissertação (Mestrado) - Escola de Enfermagem, Universidade de São Paulo. 
09. TANNENBAUM, R.; WESCHLER, J. R.; MASSARIK, F. Liderança e organização: uma abordagem à ciência do comportamento. Trad. Auriphebo B. Simões. São Paulo: Atlas, 1970.

10. TREVIZAN, M. A. Enfermagem hospitalar: administração \& burocracia. Brasília: Editora Universidade de Brasília, 1988.
11. TREVIZAN, M. A.; MENDES, I. A. C.; FÁVERO, N.; GALVÃO, C. M.; REIS, J. N. O esperado e o praticado pelo enfermeiro em relação à liderança no ambiente hospitalar: visão do atendente de enfermagem. Adm.Saúde, v.17, n.1, p.31-4, janeiro/fevereiro 1993.

12. YURA, H.; OZIMEK, D.; WALSH, M. B. Nursing leadership: theory and process. 2. ed. New York: Appleton-Century-Crofts, 1981. 